\title{
3D-Visualization and Registration for Neurovascular Compression Syndrome Analysis
}

\author{
P. Hastreiter ${ }^{1,2}$, R. Naraghi ${ }^{1}$, B. Tomandl ${ }^{3}$, M. Bauer ${ }^{2}$, and R. Fahlbusch ${ }^{1}$ \\ ${ }^{1}$ Neurocenter, Dept. of Neurosurgery, Schwabachanlage 6, 91054 Erlangen, Germany \\ hastreiter@neurocenter.imed.uni-erlangen.de \\ ${ }^{2}$ Computer Graphics Group, University of Erlangen-Nuremberg, Germany \\ ${ }^{3}$ Division of Neuroradiology, Schwabachanlage 6, 91054 Erlangen, Germany
}

\begin{abstract}
Neurovascular compression syndromes are caused by a pathological contact between vessels and the root entry or exit zone of cranial nerves. Associated with a number of neurological diseases such as trigeminal neuralgia, hemifacial spasm or vertigo, there is also strong evidence for a direct relation with essential arterial hypertension. To treat the related conditions, operative microvascular decompression has proven to be very effective. So far, as a drawback for the examination, 2D representations of tomographic volumes served as exclusive source of information. Aiming at an improved spatial understanding, we introduce a noninvasive and fast approach providing clear delineation and interactive $3 \mathrm{D}$ visualization of all relevant structures. It is based on strongly T2 weighted MR volumes with sufficiently high resolution. Due to the size of the nerves and vessels at the brainstem, an explicit segmentation is extremely difficult. Therefore, we propose to segment only coarse structures with a sequence of filtering and volume growing. Consecutively, implicit segmentation with pre-defined transfer functions is applied to delineate the tiny target structures included in the area of cerebrospinal fluid. Additionally, we suggest registration with MR angiography to differentiate between vessels and nerves on one side and between arteries and veins on the other side. Overall, our approach contributes significantly to an optimized 3D analysis of vascular compression syndromes. The high value for the planning of surgery is demonstrated with several clinical examples.
\end{abstract}

Keywords: Visualization, Registration, Neurovascular Compression

\section{Introduction}

Neurovascular compression syndromes (NVC) such as trigeminal neuralgia or hemifacial spasm characterized by hyperactive cranial nerve dysfunction result from compression of the root entry or exit zone of cranial nerves by small vessels [1] (see Fig. 1). Although the pathophysiology of neurovascular compression is still unknown, microvascular decompression has been proven to be effective and is regarded as the treatment of choice. Since the neurovascular structures are very small, imaging and consecutive visualization of neurovascular compression is extremely difficult [2].

Intraoperative observations in cases with trigeminal neuralgia or hemifacial spasm with additional arterial hypertension showed a distinct neurovascular compression of the ventrolateral medulla on the left at the level of the root entry zone of the cranial nerves IX and X [1]. Microvascular decompression of the ventrolateral medulla was 
able to reduce high blood pressure permanently in about $70 \%$ of the operated cases [3]. These observations were supported by comparative microanatomical dissections [4]. As a result, three distinct types of neurovascular compression at the ventrolateral medulla were described and the posterior inferior cerebellar artery (PICA) was identified as the predominant offending vessel. Further studies showed that operative microvascular decompression effectively contributes to reduce an abnormal elevated blood pressure[5].
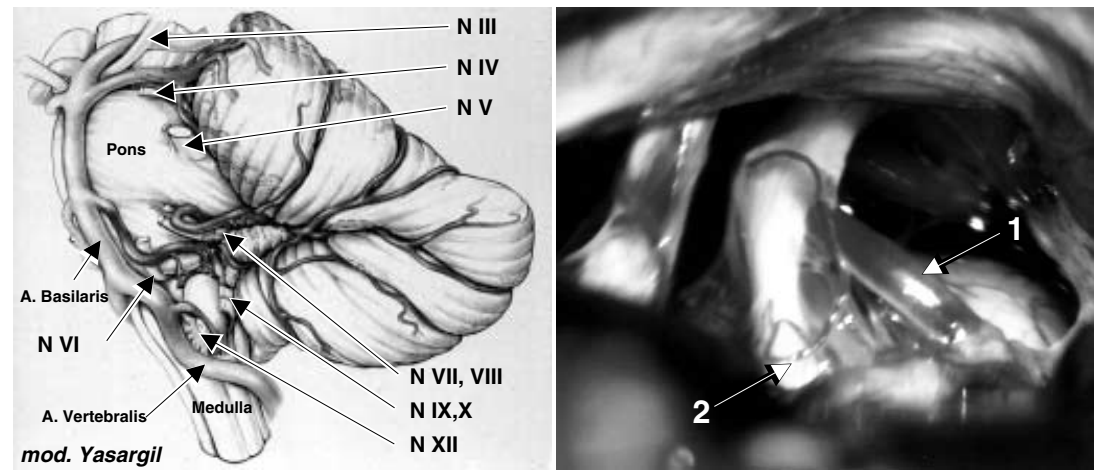

Fig. 1. Anatomical illustration (left) showing the neurovascular relations at the brainstem including the cranial nerves (III-XII) and vessels. - Intraoperative view (right) showing neurovascular compression $(1 \rightarrow)$ and trigeminal nerve with distortion of the root entry zone $(2 \rightarrow)$

A comprehensive analysis of neurovascular compression syndromes in preparation of surgery requires detailed understanding of all relevant structures. Therefore, it is important to display the relationship of the cranial nerves to the vascular structures and the brainstem. A delineation of these structures is obtained with magnetic resonance imaging (MRI). In this context the strongly T2 weighted CISS (Constructive Interference in the Steady State) sequence turned out to be most appropriate since it reveals high contrast between the vascular structures and the cerebrospinal fluid (CSF). Thereby, even small structures of less than $1 \mathrm{~mm}$ in diameter are visible. However, the 2D visualization of slice images is insufficient to precisely identify the location and relation of cranial nerves and vessels. To overcome this situation a clear and interactive $3 \mathrm{D}$ visualization is necessary contributing to an improved spatial understand.

After an overview about the image data in section 2, the applied segmentation is explained in section 3 integrating experience presented in [6]. Since an explicit segmentation of the tiny vessels and nerves is too time-consuming and error-prone, we suggest to use implicit segmentation based on direct volume rendering. Using standard PC graphics hardware [7] transfer functions for color and opacity values are interactively adjusted starting with pre-defined lookup tables. As a prerequisite this approach requires only a coarse segmentation of the image data which is conveniently obtained with a fast semi-automatic strategy. Then, section 4 outlines the evolved transfer functions and the actual visualization. Further on, voxel based registration with MR angiography (MRA) is suggested. Thereby, the differentiation between vessels and nerves in MR-CISS data is made easier and faster. Finally, section 6 discusses our approach and presents clinical examples demonstrating its value for practical application. 


\section{Image Data}

Using different scanning parameters, MRI provides enormous potential in order to differentiate a great variety of tissues non-invasively. It is the only imaging modality to scan neuronal tissue, cranial nerves and vascular structures within the posterior fossa with sufficiently high resolution. For the analysis of neurovascular compression syndromes a special imaging protocol was developed. In the beginning a MR-FLAIR (Fluid Attenuated Inversion Recovery) sequence is used which produces water suppressed T2 weighted images. Thereby, other lesions such as infarction or tumerous lesions are excluded. Consequently, a MR-CISS sequence is applied providing images with high signal of CSF and low intensity for nerves and vascular structures. Since the target structures are within the space of the CSF surrounding the brainstem, they are clearly defined as can be seen in Fig.3. However, they are in the same range of data values as the remaining soft tissues which prohibit simple maximum intensity projection $(M I P)$. In the same way, the explicit segmentation of tiny structures is very time-consuming and, on top of this, error-prone due to the extremely low resolution and partial volume effects. However, using locally applied color and opacity lookup tables exclusively, it is possible to clearly delineate all vascular structures and nerves with direct volume rendering. In addition to that it is important to provide interactive manipulation of transfer functions ensuring time-saving and accurate implicit segmentation.
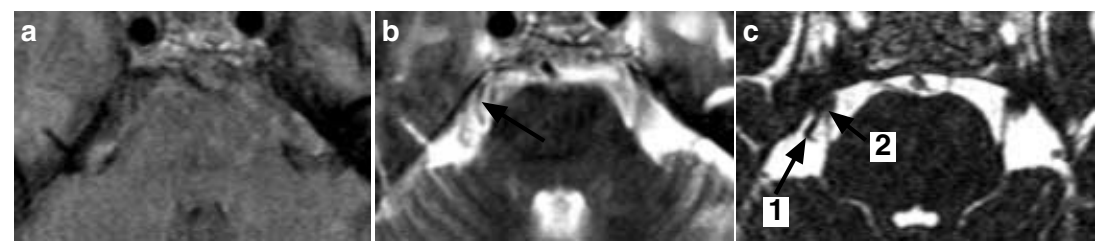

Fig. 2. Imaging of a right trigeminal neuralgia with different MR sequences: (a) no detailed anatomical structures with MR-FLAIR, (b) unreliable delineation of neurovascular relations $(\rightarrow)$ with MR-T2 due to CSF pulsation artifacts, (c) clear detection of vessels $(1 \rightarrow)$ and nerves $(2 \rightarrow)$ in CSF with MR-CISS

\section{Segmentation}

An important issue of the presented approach is the high difference of signal contrast produced for CSF and non-liquid structures using the MR-CISS sequence. As shown in section 2 the CSF is mapped to high intensity values while the enclosed target vessels and nerves are reproduced with low signal. However, applying implicit segmentation with a single lookup table for color and opacity values leads to unsatisfying visualization results. This results from identical ranges of intensity values for the target structures, the brainstem and further surrounding tissues. As a solution to this problem, we suggest to explicitly segment the essential CSF area containing all vessels and nerves. In consequence, local transfer functions provide a clear visualization if they are applied to this segmented object. 
The process of segmentation as a necessary prerequisite for a consecutive meaningful visualization subdivides into the following steps:

Noise Reduction: Due to the noisy character of the data (compare Fig. 2) it is helpful to perform a noise reduction in the beginning. For this purpose anisotropic diffusion [8] is performed providing more homogeneous areas while preserving boundaries.

Morphological Filtering: In order to simplify the actual segmentation of the CSF volume, morphological filtering with a 3D greyvalue closing operation is applied. Thereby, the vessels and nerves are removed which are represented by low intensity values within the CSF volume. Afterwards, the "closed" CSF volume forms a compact object with a clear boundary which is easily extracted. To ensure good results the applied spherical filter kernel should be smaller than surrounding structures and greater than the target vessels and nerves. For this purpose a radius of $r=2$ turned out to be optimal.

Volume Growing: The actual extraction of the CSF volume is then performed with volume growing. To make this process more robust, it is important to use bounding boxes and to proceed stepwise. Thereby, restricting the growing process to user-defined subvolumes, the integration of irrelevant neighboring structures is prevented. The segmented "closed" CSF volume is consecutively used as a mask to label the original MR-CISS data for volume rendering.

Manual Labeling: For the differentiation of vessel structures and cranial nerves within the segmented CSF volume, labeling of the nerves must be performed. This is so far achieved manually since it requires a comprehensive anatomical knowledge. Additionally, the brainstem is labeled to obtain an improved 3D representation. Since the ventral side of the brainstem is of interest, the already existing mask of the CSF volume serves as an explicit boundary for a further manual but fast labeling.

Attributing: As a final step of segmentation the original MR-CISS data is attributed (compare Fig.3) with different tags for the background (tag 0), the CSF volume including the vessels (tag 1), the cranial nerves (tag 2) and the brainstem (tag 3 ).

The suggested semi-automatic segmentation approach leads to fast pre-processing, due to the immediate visual control and the low computational expense required for the single steps. Further on, integrating the experts knowledge directly for this highly specialized task ensures robust results.

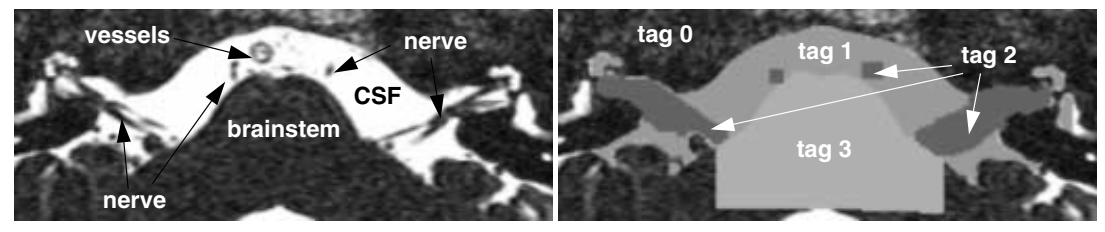

Fig. 3. MR-CISS slice showing with CSF containing tiny vascular structures and nerves (left) and coarse segmentation (right) of background (tag 0), CSF containing vessels (tag 1), CSF including nerves (tag 2), and brainstem (tag 3) 


\section{Visualization}

Having segmented and labeled the MR-CISS volume as explained in section 3, the actual delineation of the vessels and nerves is performed implicitly based on direct volume rendering using 3D texture mapping with standard PC graphics hardware. For this purpose pre-defined lookup tables for colors and opacity values are used for every subvolume (see Fig 4). They are interactively adjusted to match an individual dataset. The intensity histogram displayed within the color editor serves as supporting information. The actual volume rendering is performed with $3 \mathrm{D}$ texture mapping using standard PC graphics hardware [7].

In general, the background (tag 0 ) is made completely transparent since the most relevant information is contained in the remaining subvolumes. Only for further anatomical orientation it is made visible by using a linear ramp for the color values combined with low opacity values. The visualization of the vessels and nerves as part of the CSF subvolumes (tag 1, tag 2) is implicitly achieved using the suggested transfer functions. Considering the target structures with MR-CISS volumes low data values are mapped to high opacity and color values. Further on, a clear delineation of the tiny vessels and nerves requires to adjust a decline for the opacity. Thereby, high data values are made transparent which guarantees a smooth transition to CSF. The respective color components obtain a higher slope to intensify the impression of depth. Additionally, full opacity and cyan color is applied to the brainstem (tag 3 ) supporting the anatomical orientation.
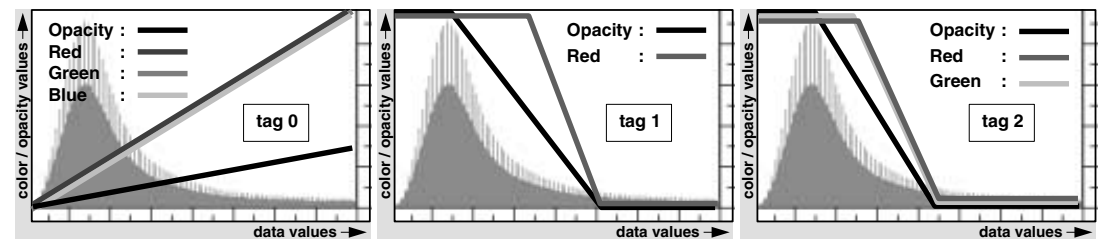

Fig. 4. Transfer functions: background (tag 0), CSF \& vessels (tag 1) and CSF \& nerves (tag 2)

\section{Fusion with MR-Angiography}

The segmented MR-CISS data allow visualizing all structures relevant for neurovascular compression syndromes in a robust way. However, as a drawback of the approach presented in section 3 manual labeling has to be performed to separate cranial nerves from vessels requiring extensive anatomical expertise. To simplify this process we introduce the integration of MR-angiography (MRA, "time of flight" (TOF)) data as additional source of vascular information in the MR-CISS volume.

An important prerequisite is the correct transformation of the MRA data to the MR-CISS coordinate system by means of registration. For this purpose, a rigid voxel based registration with mutual information [9 10] was used. To ensure fast alignment a hardware accelerate strategy was applied which was introduced in [11]. Thereby, capabilities of 3D texture mapping subsystems are used to cope with the huge amount of trilinear interpolation operations dominating the overall calculation time. 
In order to extract the vascular information we applied anisotropic diffusion for initial noise suppression and consecutive thresholding. Attempts using line filters improved our results only marginally. Thereafter, the segmented structures were copied into the MR-CISS data and served as a mask to label the respective subvolumes. Thereby, vessels are conveniently separated from nerves and arteries are differentiated from veins. Furthermore, vascular structures at the boundary layer of the CSF are delineated in a more robust way.

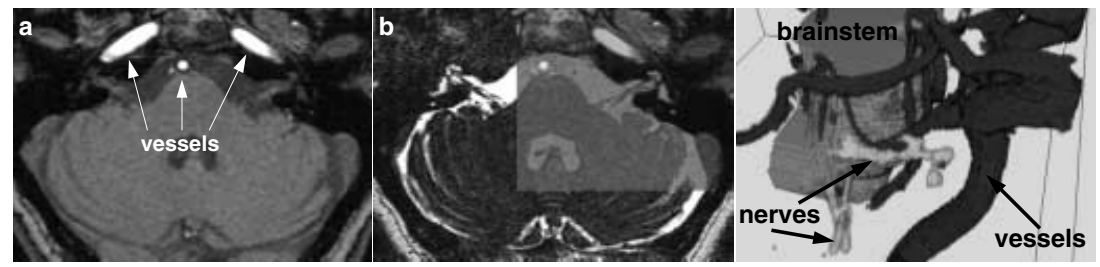

Fig. 5. Fusion of MR-CISS and MRA-TOF: (1) vessels within MRA, (2) overlay of MR-CISS and MRA using magic lens, (3) 3D visualization from MR-CISS using vessel mask from MRA

\section{Results and Discussion}

The presented approach was so far applied to 27 patients. Out of this group, 19 suffered from trigeminal neuralgia, 4 from hemifacial spasm and 4 from hypertension. Surgery was conducted in 23 cases. In all cases, MR-FLAIR, -T2, -TOF and -CISS data were acquired. All volumes were scanned with a Siemens MR Magnetom Sonata 1.5 Tesla scanner. In all cases the MR-CISS and -TOF data consisted of $384 \times 512 \times 62-128$ voxels with an average size of $0.39 \times 0.39 \times 0.7 \mathrm{~mm}^{3}$. In case of MR-FAIR and MR-T2 volumes of $408 \times 512 \times 23$ voxels with a size of $0.45 \times 0.45 \times 6.0 \mathrm{~mm}^{3}$ were obtained.

A standard PC (AMD Athlon 1.2 GHz) with NVidia GeForce3 graphics card providing $64 \mathrm{MB}$ texture memory was used for the interactive direct volume rendering based on $3 \mathrm{D}$ texture mapping. In order to find the optimal setting for the transfer functions only a few and simple operations were necessary. The same platform was also applied for the registration requiring $4.5 \mathrm{~min}$ for the alignment. Thereby, a single interpolation of the volume including the evaluation of mutual information requires only $0.73 \mathrm{sec}$ using a viewport of $150 \times 150$ pixels.

To our knowledge the presented approach produced for the first time meaningful $3 \mathrm{D}$ visualizations for the analysis of neurovascular compression syndrome based on MR-CISS data. In all cases, the brainstem, the small vessels (PICA, AICA, SCA) and the cranial nerves were identified. Two selected cases demonstrate the clinical value of our strategy.

Case 1: In Fig. 6 the situation of a trigeminal neuralgia is illustrated. The 3D representations clearly show the relation of the relevant vessels and nerves confirmed by operative findings. For the same case the masking of vascular structures was also performed with MRA-TOF as shown in Fig. 5 In this way the bigger vessels are clearly delineated while the small vessels are problematic due to reduced flow signal. Consequently, MR-CISS remains indispensable to detect all relevant structures but MRA-TOF is a good supplement to simplify labeling. 
Case 2: Fig. 7 presents the neurovascular anatomy in arterial hypertension. In this case a close relationship of the PICA-loop to the left ventrolateral medulla at the root entry zone of the cranial nerves IX and $X$ as type $1[4]$ is visible.

At the current stage, the overall time for a 3D analysis of neurovascular compression syndromes requires about 2 hours. It consists of MR imaging ( $25 \mathrm{~min}$ ), complete explicit segmentation (1 hours), registration (4.5 min) and visualization (5 min). Thereby, most of the time is still consumed for labeling the MR-CISS data. However, this step provides further potential for optimization.

\section{Conclusion}

A non-invasive approach was introduced allowing for a comprehensive 3D analysis of neurovascular compression syndromes. It clearly shows the relation of all relevant structures implicitly delineated from MR data after only a coarse explicit segmentation. Overall, this strategy is robust and comparatively fast. For the future a fully automatic strategy for the differentiation of vessels and nerves is envisaged. Resulting from comparisons with operative findings our method is of high value for preoperative evaluation and contributes to optimize clinical practice.

\section{References}

1. P. Jannetta. Neurovascular compression in cranial nerve and systemic disease. Ann. Surg., 192:518-525, 1980.

2. R. Naraghi, H. Geiger, J. Crnac, W. Huk, R. Fahlbusch, G. Engels, and F. Luft. Posterior fossa neurovascular anomalies in essential hypertension. The Lancet, 344:1466-1470, 1994.

3. P. Jannetta, R. Segal, and S. Jr. Wolfson. Neurogenic hypertension: etiology and surgical treatment. I. Observations in 53 patients. Ann. Surg., 201:391-398, 1985.

4. R. Naraghi, M. Gaab, G. Walter, and B. Kleineberg. Arterial hypertension and neurovascular compression at the ventrolateral medulla. A comparative microana-tomical and pathological study. J. Neurosurg., 77:103-112, 1992.

5. H. Geiger, R. Naraghi, H.P. Schobel, H. Frank, R.B. Sterzel, and R. Fahlbusch. Decrease of blood pressure by ventrolateral medullary decompression in essential hypertension. The Lancet, 352:446-449, 1998.

6. C. Rezk-Salama, P. Hastreiter, K. Eberhardt, B. Tomandl, and T. Ertl. Interactive Direct Volume Rendering of Dural Arteriovenous Fistulae. In Proc. MICCAI, Lect. Notes in Comp. Sc., pages 42-51. Springer, 1999.

7. C. Rezk-Salama, K. Engel, M. Bauer, G. Greiner, and T. Ertl. Interactive Volume Rendering on Standard PC Graphics Hardware Using Multi-Textures and Multi-Stage Rasterization. In Proc. Eurographics/SIGGRAPH Workshop on Graphics Hardware, 2000.

8. G. Gerig, O. Kübler, R. Kikinis, and F. Jolesz. Nonlinear Anisotropic Filtering of MRI Data. IEEE Trans. on Med. Imag., 11(2):221-232, 1992.

9. P. Viola and W. Wells. Alignment by Maximization of Mutual Information. In Proc. Vth Int. Conf. Comp. Vision, pages 16-23, Cambridge, MA, 1995.

10. A. Collignon, D. Vandermeulen, P. Suetens, and G. Marchal. Automated Multi-Modality Image Registration Based on Information Theory. Kluwen Acad. Publ's: Comput. Imag. and Vis., 3:263-274, 1995.

11. P. Hastreiter and T. Ertl. Integrated Registration and Visualization of Medical Image Data. In Proc. CGI, pages 78-85, Hannover, Germany, 1998. 

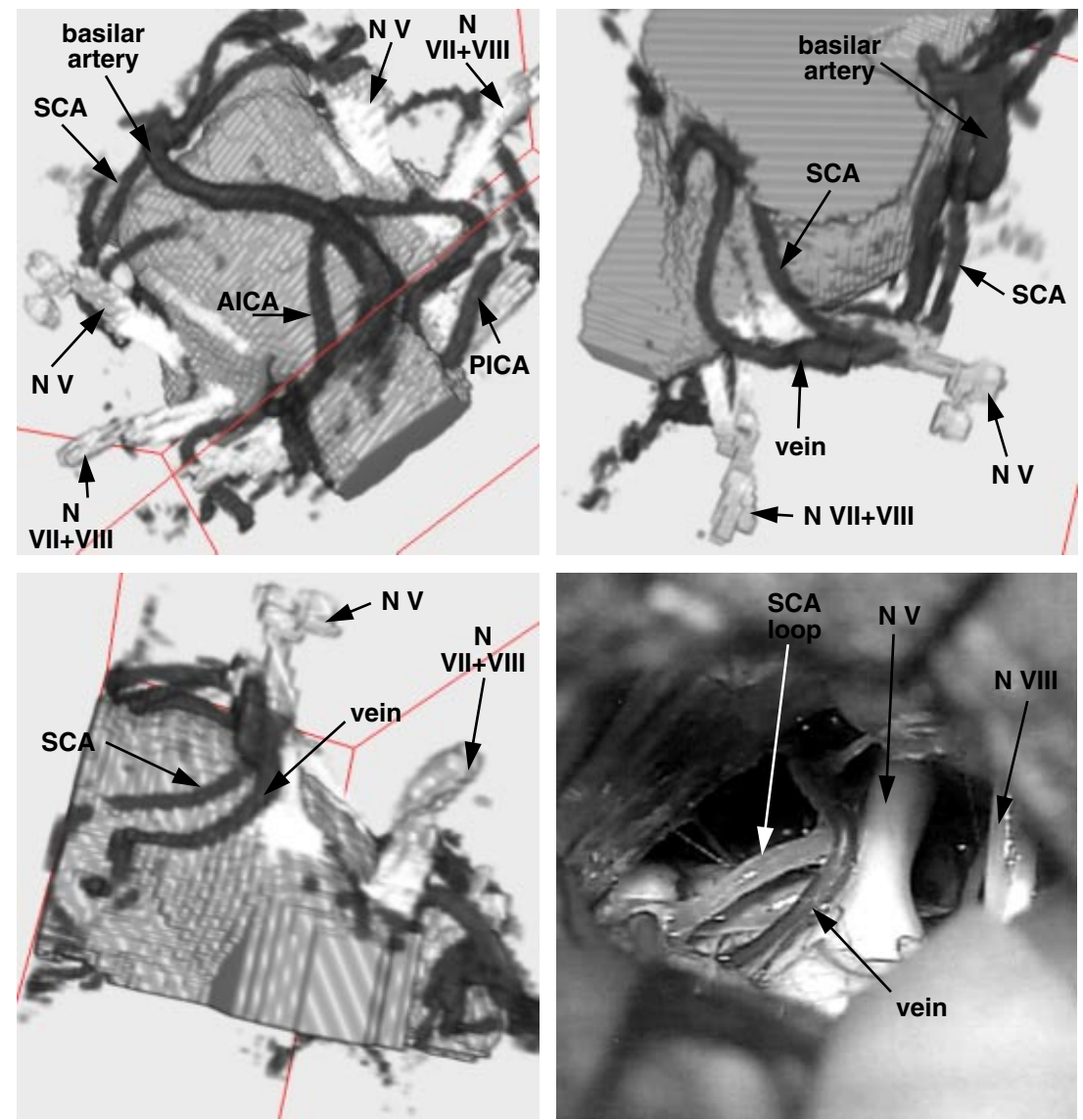

Fig. 6. Visualization of NVC in trigeminal neuralgia showing relations between vessels and nerves (top row). Visualization compared to operative finding (bottom row)
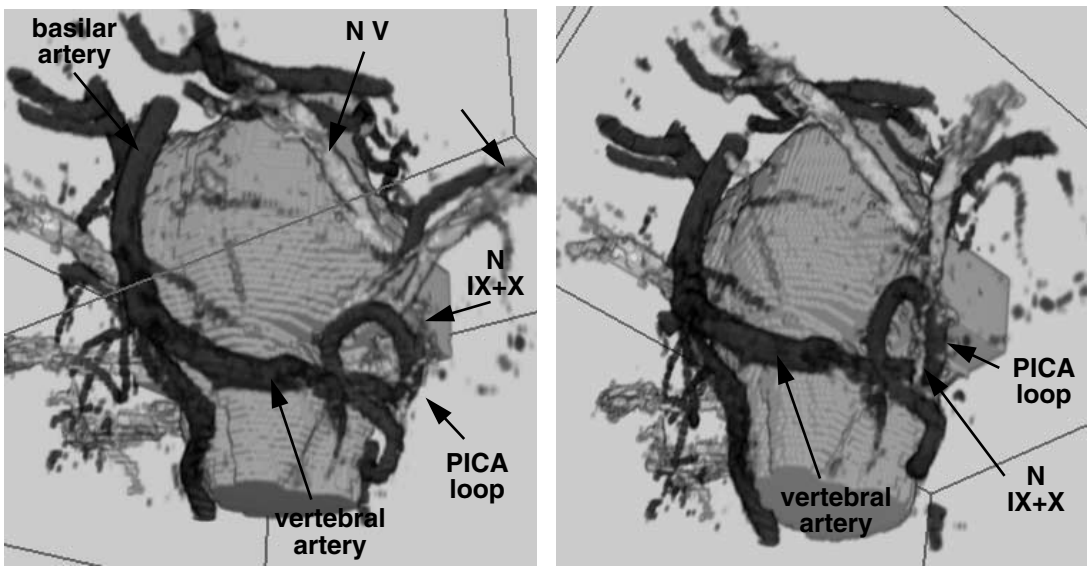

Fig. 7. Visualization of NVC at the ventrolateral medulla in essential hypertension, looping PICA 